05

\title{
Возможности магнитного псевдорезонанса в изучении тонких ферромагнитных пленок с одноосной магнитной анизотропией
}

\author{
(C) В.В. Демидов, ${ }^{1}$ A.E. Мефёд ${ }^{2}$ \\ ${ }^{1}$ Институт радиотехники и электроники им. В.А. Котельникова РАН, \\ 125009 Москва, Россия \\ ${ }^{2}$ Институт радиотехники и электроники им. В.А. Котельникова РАН, Фрязинский фрилиал, \\ 141190 Фрязино, Московская обл., Россия \\ e-mail: demidov@cplire.ru
}

(Поступило в Редакцию 8 февраля 2018 г.)

Выяснены возможности магнитного псевдорезонанса (нерезонансного пика магнитной восприимчивости) в сравнении с ферромагнитным резонансом (ФМР) при измерении параметров тонких ферромагнитных пленок с плоскостной одноосной магнитной анизотропией. Измерения проведены на двух характерных для данного эффекта образцах ферромагнитных пленок. Использовались $Q$-метр на частоте около $300 \mathrm{MHz}$ (для псевдорезонанса) и стандартный магнитно-резонансный спектрометр $X$-диапазона (для ФМР). Показано, что техника $Q$-метра на частоте $300 \mathrm{MHz}$ обеспечивает адекватную регистрацию магнитного псевдорезонанса как в эпитаксиальных, так и в поликристаллических пленках. Установлено, что по точности определения величины поля магнитной анизотропии и направления легкой оси намагничивания метод псевдорезонанса не уступает ФМР, а в некоторых случаях информативно дополняет его.

DOI: $10.21883 /$ JTF.2019.01.46965.55-18

В последние десятилетия тонкие анизотропные ферромагнитные пленки вызывают повышенный интерес из-за перспективы их широкого практического использования. Особое внимание привлекают пленки с одноосной плоскостной магнитной анизотропией. Традиционно магнитную анизотропию в пленках изучают с помощью вибрационного магнетометра, магнитооптического эффекта Керра и ферромагнитного резонанса (ФМР). Последний метод дает наиболее полную информацию о величине, симметрии и направлении внутренних магнитных полей в ферромагнетиках. Недавно в ферромагнитных пленках с плоскостной одноосной анизотропией магнитного поля был обнаружен эффект резкого усиления радиочастотной (РЧ) динамической магнитной восприимчивости [1-3]. Он наблюдается, когда внешнее магнитное поле Н лежит в плоскости пленки, направлено перпендикулярно ее оси легкого намагничивания $\mathbf{n}_{u}$ (и, следовательно, параллельно трудной оси) и проходит через значение $H=H_{u}$, где $H_{u}-$ поле одноосной внутриплоскостной магнитной анизотропии пленки. При этом регистрирующее радиочастотное магнитное поле $\mathbf{h} e^{i \omega t}$ направлено перпендикулярно полю $\mathbf{H}$, а его частота $\omega$ фиксирована. Эффект проявляется в виде сравнительно узкого резонансно-подобного сигнала поглощения с максимумом при значении поля $H=H_{0}$, которое в данном случае равно $H_{u}$. Особенностью этого сигнала является то, что при установке другой частоты $\omega$ в широких пределах величина поля, при котором он наблюдается, остается неизменной и равной $H_{0}$. Этот эффект, названный „магнитным псевдорезонансом“ [3], обусловлен тем, что при достижении полем $H$ значения $H_{u}$ происходит резкий самопроизвольный поворот равновесной намагниченности образца $\mathbf{M}_{0}$ от исходного направления $\mathbf{n}_{u}$ к направлению внешнего поля $H$, совпадающему с направлением трудной оси пленки. При этом соответственно возрастает поперечная относительно поля Н магнитная восприимчивость образца, приводящая к пику РЧ поглощения. В работе [3] этот эффект был обнаружен с помощью $Q$-метра, работавшего на частоте около $300 \mathrm{MHz}$ [4]. $Q$-метр такого частотного диапазона оказался весьма подходящим инструментом для исследования псевдорезонанса в ферромагнитных пленках с одноосной магнитной анизотропией, поскольку он достаточно чувствителен, доступен (так как легко может быть изготовлен в лабораторных условиях), удобен и надежен в эксплуатации. Ниже описаны характерные примеры использования $Q$-метра для регистрации псевдорезонанса в анизотропных ферромагнитных пленках и изучения их магнитных свойств. Измеренные при этом параметры анизотропии сравниваются с данными, полученными на тех же образцах с помощью ФМР на частоте $9.6 \mathrm{GHz}$.

Исследования псевдорезонанса проводились при комнатной температуре с помощью $Q$-метра, работающего на частоте $296 \mathrm{MHz}$ [4]. Одним из образцов была эпитаксиальная пленка $\mathrm{La}_{2 / 3} \mathrm{Sr}_{1 / 3} \mathrm{MnO}_{3}$ (LSMO) толщиной $50 \mathrm{~nm}$, полученная путем напыления на плоскость (110) монокристаллической подложки из $\mathrm{NdGaO}_{3}$ размером $5 \times 5 \times 0.5 \mathrm{~mm}^{3}[3,5]$. При комнатной температуре пленка находилась в ферромагнитном состоянии (ее температура Кюри $\left.T_{C} \approx 355 \mathrm{~K}\right)$. Кристаллические параметры пленки и подложки несколько отличаются друг от друга, что вызывает механические напряжения с формированием одноосной магнитной анизотропии в плоскости пленки. Величина поля анизотропии $\left(H_{u}=206.2 \pm 0.8\right.$ Ое $)$ и направление оси $\mathbf{n}_{u}$, которая оказалась параллельной двум сторонам подложки, вначале были определенны 


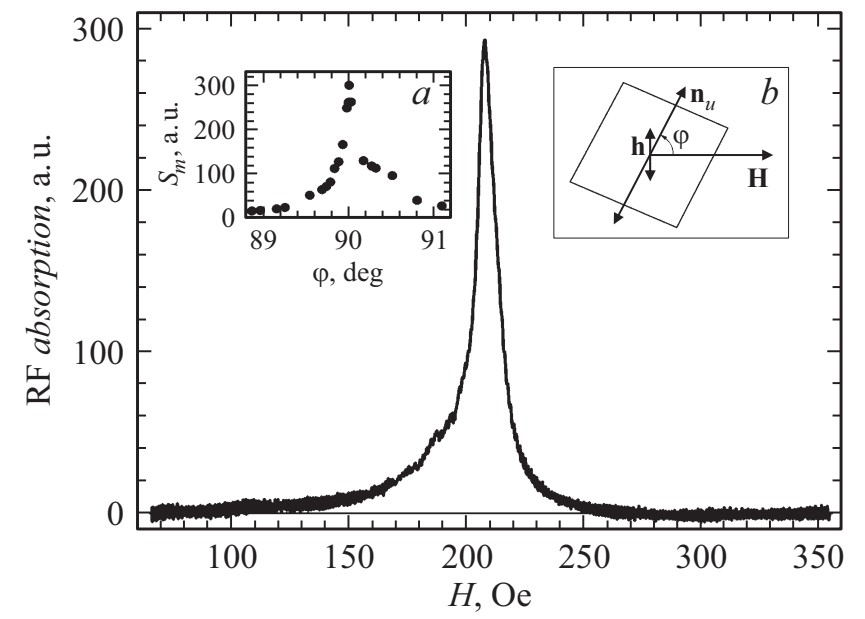

Рис. 1. Сигнал РЧ поглощения (псевдорезонанс) от одноосной ферромагнитной пленки LSMO при развертке внешнего магнитного поля $H$ через значение $H_{u}=207.1$ Ое в условиях $\mathbf{H} \perp \mathbf{n}_{u}$ и $\mathbf{h} \| \mathbf{n}_{u}(\varphi=\pi / 2)$. Вставка $a-$ зависимость пикового значения $S_{m}$ сигнала псевдорезонанса от угла $\varphi$ при $H=207.1 \mathrm{Oе}, b-$ схема взаимной ориентации поля $\mathbf{H}$, РЧ поля $\mathbf{h}$ и легкой оси $\mathbf{n}_{u}$, находящихся в плоскости пленки (квадрат).

из угловой зависимости спектра ФМР, снятого на стандартном спектрометре ЭПР ER 200 фирмы Bruker с частотой $9.6 \mathrm{GHz}$ [5]. При исследовании методом $Q$-метра подложка с пленкой помещалась в центр катушки индуктивности датчика и с помощью гониометра с двумя взаимно перпендикулярными вращениями (с точностью $\pm 0.1^{\circ}$ ) ориентировалась так, что оба поля, Н и $\mathbf{h}$, лежали в плоскости пленки. Величина угла $\varphi$ между осью $\mathbf{n}_{u}$, лежащей в плоскости пленки, и полем $\mathbf{H}$ устанавливалась путем врашения образца вокруг оси, перпендикулярной плоскости пленки. Поиск сигнала псевдорезонанса осуществлялся путем треугольной развертки поля $H$ от нуля до нескольких сотен эрстед и обратно с периодом $200 \mathrm{~s}$, при ступенчатом изменении угла $\varphi$ с шагом $0.3^{\circ}$. Амплитуда РЧ поля $h$ была порядка 0.002 Ое. Сигнал обрабатывался амплитудным детектором и регистрировался на постоянном токе методом выборок с шагом около $0.1 \mathrm{~s}$, т.е. без использования дополнительной модуляции поля Н и синхронного детектирования. Для улучшения отношения сигнал/шум проводилось накопление сигнала.

Вид полученного псевдорезонансного сигнала поглощения при $\varphi=90^{\circ}\left(\mathbf{H} \perp \mathbf{n}_{u}\right)$ и девятикратном его накоплении показан на рис. 1. На вставке $b$ показана взаимная ориентация векторов полей $\mathbf{H}, \mathbf{h}$ и оси $\mathbf{n}_{u}$; все они находятся в плоскости пленки. Максимуму сигнала соответствует поле $H_{0}=207.1 \pm 0.4$ Ое. В пределах погрешности измерений оно совпадает по величине с приведенным выше полем $H_{u}$, измеренным методом ФМР на частоте $9.6 \mathrm{GHz}$.

На вставке $a$ на рис. 1 показана зависимость пиковой величины $S_{m}$ сигнала псевдорезонанса от угла $\varphi$ между легкой осью $\mathbf{n}_{u}$ и полем Н. Видно, что эта зависимость также имеет резонансный вид, причем пику соответствует угол $\varphi=90 \pm 0.1^{\circ}$, а ширина на полувысоте составляет $0.2^{\circ}$. Видно также, что при отходе от угла $\varphi=90^{\circ}$ всего на $\pm 1^{\circ}$ сигнал псевдорезонанса уменьшается настолько, что становится практически ненаблюдаемым. Это говорит о весьма точном определении направления оси $\mathbf{n}_{u}$ в пленке. Оно совпадает с направлением, полученным из анализа спектра ФМР на частоте $9.6 \mathrm{GHz}$.

Таким образом, методом магнитного псевдорезонанca, регистрируемого с помощью $Q$-метра на частоте $\sim 300 \mathrm{MHz}$, в одноосных ферромагнитных пленках можно определить величину поля анизотропии $H_{u}$ и ориентацию легкой оси намагничивания $\mathbf{n}_{u}$ практически с той же точностью, что и методом ФМР на $9.6 \mathrm{GHz}$. Однако возможности магнитного псевдорезонанса этим не исчерпываются. В следующем примере мы покажем, что на практике могут встречаться случаи, когда он может дать полезную дополнительную информацию, недоступную методу ФМР.

Таким примером может служить гетероструктура в виде „сэндвича“ $\left(\mathrm{FeCo} / \mathrm{TbCo}_{2} / \mathrm{FeCo}\right) / \mathrm{MgO} /\left(\mathrm{FeCo} / \mathrm{TbCo}_{2} /\right.$ $\mathrm{FeCo})^{1}$, перспективная для создания магнитно-управляемых коммутаторов [6]. Две его обкладки (в скобках) представляют собой трехслойные поликристаллические пленки из интерметаллидов с толщинами слоев $2 \mathrm{~nm}$ $(\mathrm{FeCo})$ и $6 \mathrm{~nm}\left(\mathrm{TbCo}_{2}\right)$, которые разделены тонкой прослойкой из $\mathrm{MgO}$ толщиной $3 \mathrm{~nm}$. Эта структура наносилась на монокристаллическую подложку из $\mathrm{NdGaO}_{3}$ с размерами $5 \times 5 \times 0.5 \mathrm{~mm}^{3}$. Обе интерметаллические пленки при комнатной температуре являются ферромагнетиками с гигантской магнитострикцией. При их напылении параллельно плоскости подложки накладывалось внешнее магнитное поле, величина которого для пленок была существенно разной, а направление одинаковым. При этом ожидалось, что легкие оси $\mathbf{n}_{u}$ магнитной анизотропии пленок будут лежать в их плоскости и будут ориентированы вдоль наложенного магнитного поля, а величины полей их магнитной анизотропии будут существенно разными.

Для поиска сигнала псевдорезонанса эта поликристаллическая пленочная структура помещалась в катушку датчика $Q$-метра, как показано на вставке $b$ к рис. 1. Сигнал регистрировался методом синхронного детектирования при двунаправленной развертке поля $H$, дополнительно модулированного с частотой $52.3 \mathrm{kHz}$ и амплитудой 1.3 Ое. Такой метод регистрации позволял при весьма малой толщине пленок получить нужное отношение сигнал/шум за приемлемое время. Регистрация велась непрерывно при многократном накоплении (до 100 раз). Полученный сигнал показан на рис. 2. Ясно видны две компоненты, соответствующие двум сигналам псевдорезонанса. Отметим, что форма этих сигналов

\footnotetext{
${ }^{1}$ Образец был изготовлен в International Associated Laboratory LEMAC-LICS:IEMN, UMR CNRS 8520, PRES Lille Nord de France, Франция.
} 


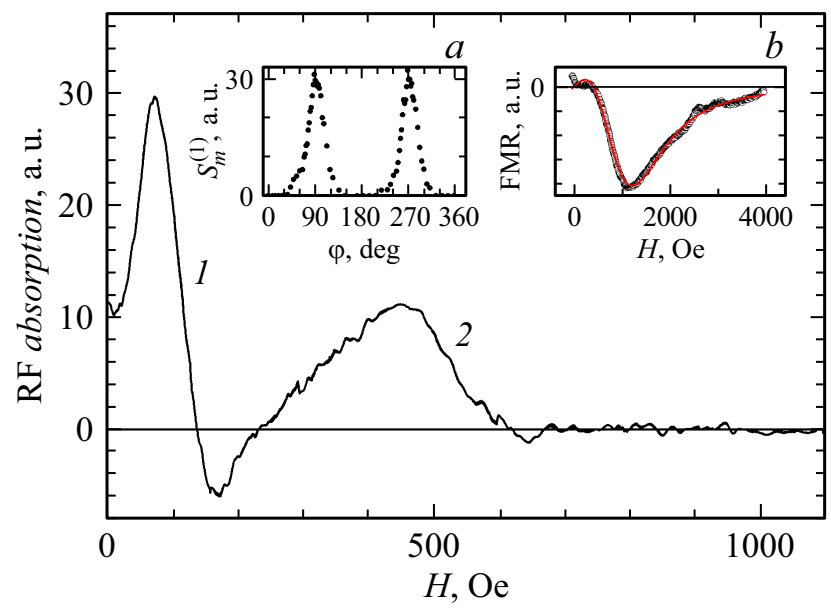

Рис. 2. Радиочастотное поглощение в пленочной ферромагнитной гетероструктуре $\left(\mathrm{FeCo} / \mathrm{TbCo}_{2} / \mathrm{FeCo}\right) / \mathrm{MgO} /$ $\left(\mathrm{FeCo} / \mathrm{TbCo}_{2} / \mathrm{FeCo}\right)$, состоящей из двух трехслойных пленок (в скобках), разделенных прослойкой из $\mathrm{MgO}$, в зависимости от величины внешнего магнитного поля $\mathbf{H}$, ориентированного перпендикулярно оси легкого намагничивания $\mathbf{n}_{u}\left(\varphi=90^{\circ}, \mathrm{cm}\right.$. вставку $b$ на рис. 1). Вставка $a-$ зависимость максимума $S_{m}^{(1)}$ сигнала 1 от угла $\varphi$ при $H_{0}=74$ Ое. Вставка $b-$ сигнал ФМР для этой гетероструктуры, полученный на частоте $9.78 \mathrm{GHz}$; сплошная кривая - производная лоренциана с параметрами, приведенными в тексте.

заметно отличается от традиционной для ЭПР спектроскопии производной поглощения по магнитному полю и больше похожа на саму линию поглощения. Искажения обусловлены особенностями явления псевдорезонанса, а также высокой чувствительностью формы сигнала псевдорезонанса при синхронном детектировании к ориентации модулирующего поля, которое в наших условиях имеет небольшую поперечную составляющую (около $10 \%$ от продольной составляющей). Численное моделирование сигнала псевдорезонанса в этих условиях с использованием уравнения Ландау-Лифшица, описывающего движение намагниченности в анизотропном ферромагнетике [7], а также экспериментальное сравнение полевого положения максимумов сигнала при прямом и синхронном методах регистрации, проведенные на эпитаксиальной пленке LSMO, показали, что они слегка различаются. Однако смещение второго относительно первого не превышает нескольких эрстед.

Следовательно, две наблюдаемые компоненты спектра РЧ поглощения (рис. 2) соответствуют двум сигналам псевдорезонанса 1 и 2, с максимумами при $H_{0}^{*}$ около 74 и 456 Ое соответственно. Здесь введено иное обозначение поля $H$ для максимума псевдорезонанса, так как между пленками „сэндвича“ существуют межслойные обменные взаимодействия [8], которые приводят к перенормировке величины поля магнитной анизотропии, делая его отличным от значения $H_{u}$ для единичного ферромагнитного слоя. Весьма разные величины $H_{0}^{*}$ у этих сигналов означают, что поля одноосной магнитной анизотропии у пленок сэндвича существенно различаются, как и было предусмотрено при изготовлении этой гетероструктуры. Для сигнала 1 была снята зависимость $S_{m}^{(1)}(\varphi)$ его максимума от угла $\varphi$ (вставка $\left.a\right)$. Она получена при развертке поля $H$ в сравнительно узком диапазоне 0-140 Ое при углах $\varphi$, задаваемых как параметр и включающих значения $\varphi=90$ и $270^{\circ}$ (вставка $b$ на рис. 1). Видно, что в обоих случаях зависимости практически одинаковы. Они оказываются значительно более пологими, чем подобная зависимость для пленки LSMO (рис. 1): их ширина на полувысоте достигает 40․ Это объясняется тем, что все слои в каждой пленке сэндвича имеют поликристаллическую структуру со значительным разбросом направления оси $\mathbf{n}_{u}$ для разных кристаллитов. Вследствие этого усредненное направление оси трудного намагничивания для пленки 1 соответствует углу $\varphi=90^{\circ}$ с точностью $\pm 4^{\circ}$. Подобные измерения для пленки 2 с более широкой линией псевдорезонанса дали значение $\varphi=90 \pm 12^{\circ}$. Отсюда следует, что в обеих пленках направления соответствующих осей внутриплоскостной магнитной анизотропии практически совпадают.

Естественно сравнить полученные данные для описанной гетероструктуры с тем, что дает метод ФМР. На вставке $b$ к рис. 2 показан сигнал ФМР от этой структуры, представляющий собой производную сигнала поглощения ФМР на частоте $9.78 \mathrm{GHz}$. Он получен методом синхронного детектирования при развертке поля $H$ в условиях его модуляции с частотой $100 \mathrm{kHz}$ и амплитудой 10 Ое с использованием 4-кратного накопления. Видно, что сигнал весьма широк, в нем нет разрешенной структуры, соответствующей двум пленкам с существенно разными величинами поля анизотропии. Экспериментально ее не удалось выявить при всех возможных ориентациях образца в поле $H$. Ее также не удалось обнаружить, моделируя сигнал суммой двух линий с формой, характерной для ФМР в условиях нашего эксперимента. В то же время этот сигнал хорошо описывается одним лоренцианом с шириной на полувысоте $\Delta_{1 / 2}=1024 \pm 6$ Ое и резонансным полем $H_{0}=675 \pm 2$ Ое (сплошная кривая на вставке $b$ ), которая не выходит за пределы контура линии ФМР. Непривычная резко асимметричная форма этого спектра объясняется тем, что резонанс наблюдается в низких полях $\left(H \sim \Delta_{1 / 2}\right)$, когда эффективны обе вращательные компоненты высокочастотного поля [9].

Возвращаясь к спектру псевдорезонанса (рис. 2), еще раз подчеркнем, что он состоит из двух хорошо разрешенных сигналов, соответствующих разным величинам поля магнитной анизотропии в пленках. Кроме того, из сигналов на вставке $a$ к рис. 2 определяется также и направление оси $n_{u}$, что в данном случае не удается сделать с помощью регистрации спектра ФМР. Это говорит о том, что магнитный псевдорезонанс, регистрируемый на частоте сотен мегагерц, может быть полезным дополнением к ферромагнитному резонансу в СВЧ диапазоне 
при исследовании тонких ферромагнитных пленок с одноосной плоскостной магнитной анизотропией.

Таким образом, магнитный псевдорезонанс позволяет успешно изучать магнитные свойства тонких ферромагнитных пленок с одноосной плоскостной магнитной анизотропией, дополняя в некоторых случаях возможности метода ферромагнитного резонанса. При этом для его адекватной регистрации подходят сравнительно простые и доступные средства, базирующиеся на технике $Q$-метра в частотном диапазоне сотен мегагерц. Отметим, что здесь впервые описан разрешенный спектр псевдорезонанса в многослойных поликристаллических пленках.

Авторы благодарят N. Tiercelin и P. Pernod из International Associated Laboratory LEMAC (France) за предоставление для исследований пленочной гетероструктуры из интерметаллидов, Г.А. Овсянникова и И.В. Борисенко за предоставление эпитаксиальной пленки LSMO, а также профессора В.А. Ацаркина за критическое обсуждение результатов работы и ценные замечания.

Работа выполнена по программе президиума РАН № 5 ,Электронный спиновый резонанс, спин-зависящие электронные эффекты и спиновые технологии“ с частичной поддержкой РФФИ (гранты № 17-02-00145 и № 18-57-16001).

\section{Список литературы}

[1] Беляев Б.А., Изотов А.В., Кипарисов С.Я. // Письма в ЖЭТФ. 2001. Т. 74. Вып. 4. С. 248-252. [Belyaev B.A., Izotov A.V., Kiparisov S.Ya. // J. Exp. Theor. Phys. Lett. 2001. Vol. 74. N 4. P. 226-230.]

[2] Василевская Т.М., Семенцов Д.И. // ЖЭТФ. 2010. T. 137. Вып. 5. С. 861-866. [Vasilevskaya T.M., Sementsov D.I. // JETP. 2010. Vol. 110. N 5. P. 754-758. DOI: $10.1134 / \mathrm{S} 1063776110050043]$

[3] Atsarkin V.A., Demidov V.V., Mefed A.E., Nagorkin V.Yu. // Appl. Magn. Reson. 2014. Vol. 45. P. 809-816. DOI: $10.1007 / \mathrm{s} 00723-014-0554-2$

[4] Мефёд А.Е., Демидов В.В. // ПТЭ. 2008. № 3. С. 99-101. [Mefed A.E., Demidov V.V. // Instr. Exp. Tech. 2008. Vol. 51. N 3. P. 418-420. DOI: 10.1134/S0020441208030160]

[5] Демидов В.В., Борисенко И.В., Климов А.А., Овсянников Г.А., Петржик А.М., Никитов С.А. // ЖЭТФ. 2011. T. 139. Вып. 5. С. 943-951. [Demidov V.V., Borisenko I.V., Klimov A.A., Ovsyannikov G.A., Petrzhik A.M., Nikitov S.A. // JETP. 2011. Vol. 112. N 5. P. 825-832. DOI: $10.1134 / \mathrm{S} 1063776111040029]$

[6] Борисенко И.В., Демидов В.В., Климов А.А., Овсянников Г.А., Константинян К.И., Никитов С.А., Преображенский В.Л., Tiercelin N., Pernod P. // Письма в ЖТФ. 2016. Т. 42. Вып. 3. C. 1-10. [Borisenko I.V., Demidov V.V., Klimov A.A., Ovsyannikov G.A., Konstantinyan K.I., Nikitov S.A., Preobrazhenskii V.L., Tiercelin N., Pernod P. // Tech. Phys. Lett. 2016. Vol. 42. N 2. P. 1-10. DOI: $10.1134 / \mathrm{S} 1063785016020048]$
[7] Гуревич А.Г., Мелков Г.А. Магнитные колебания и волны. М.: Наука, 1994. § 2.1. 464 с. [Gurevich A.G., Melkov G.A. Magnetization Oscillations and Waves. N. Y.: CRC, 1996. § 2.1. 464 p.]

[8] Дровосеков А.Б., Жотикова О.В., Крейнес Н.М., Мешеряков В.Ф., Миляев М.А., Ромашев Л.Н., Устинов В.В., Холин Д.И. // ЖЭТФ. 1999. Т. 116. Вып. 5. C. 1817-1833. [Drovosekov A.B., Zhotikova O.V., Kreines N.M., Meshcheryakov V.F., Milyaev M.A., Romashev L.N., Ustinov V.V., Kholin D.I. // JETP. 1999. Vol. 89. N 5. P. 986-994.]

[9] Альтшуллер С.А., Козырев Б.М. Электронный парамагнитный резонанс. М.: Физматлит, 1961, § 1.4. 368 с. [Al'shuler S.A., Kozyrev B.M. Electron Paramagnetic

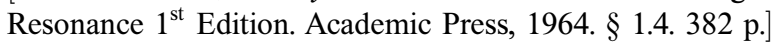

